\title{
H/D exchange mass spectrometry and statistical coupling analysis reveal a role for allostery in a ferredoxin-dependent bifurcating transhydrogenase catalytic cycle
}

\author{
Authors: Luke Berry, Saroj Poudel, Monika Tokmina-Lukaszewska, \\ Daniel R. Colman, Diep M. N. Nguyen, Gerrit J. Schut, Michael \\ W.W. Adams, John W. Peters, Eric S. Boyd, \& Brian Bothner
}

NOTICE: this is the author's version of a work that was accepted for publication in BBA - General Subjects. Changes resulting from the publishing process, such as peer review, editing, corrections, structural formatting, and other quality control mechanisms may not be reflected in this document. Changes may have been made to this work since it was submitted for publication. A definitive version was subsequently published in BBA - General Subjects, VOL\# 1862, ISSUE\# 1, (January 2018). DOI\#10.1016/j.bbagen.2017.10.002

Berry, Luke, S Poudel, M Tokmina-Lukaszewska, D.R. Colman, D.M.N. Nguyen, G.J. Schut, M.W.W. Adams, J.W. Peters, Eric S. Boyd, and Brian Bothner. "H/D exchange mass spectrometry and statistical coupling analysis reveal a role for allostery in a ferredoxin-dependent bifurcating transhydrogenase catalytic cycle." BBA - Biochimica et Biophysica Acta 1862, no. 1 (January 2018): 9-17. DOI: 10.1016/j.bbagen.2017.10.002.

Made available through Montana State University's ScholarWorks scholarworks. montana.edu 


\title{
H/D exchange mass spectrometry and statistical coupling analysis reveal a role for allostery in a ferredoxin-dependent bifurcating transhydrogenase catalytic cycle
}

\author{
Luke Berry $^{\mathrm{a}}$, Saroj Poudel ${ }^{\mathrm{a}}$, Monika Tokmina-Lukaszewska ${ }^{\mathrm{a}}$, Daniel R. Colman ${ }^{\mathrm{a}}$, \\ Diep M.N. Nguyen ${ }^{\mathrm{b}}$, Gerrit J. Schut ${ }^{\mathrm{b}}$, Michael W.W. Adams ${ }^{\mathrm{b}}$, John W. Peters ${ }^{\mathrm{c}}$, Eric S. Boyd ${ }^{\mathrm{a}}$, \\ Brian Bothner,
}

a Montana State University, Bozeman, MT 59717, United States
b University of Georgia, Athens, GA 30602, United States

${ }^{c}$ Washington State University, Pullman, WA, United States

\begin{abstract}
Recent investigations into ferredoxin-dependent transhydrogenases, a class of enzymes responsible for electron transport, have highlighted the biological importance of flavin-based electron bifurcation (FBEB). FBEB generates biomolecules with very low reduction potential by coupling the oxidation of an electron donor with intermediate potential to the reduction of high and low potential molecules. Bifurcating systems can generate biomolecules with very low reduction potentials, such as reduced ferredoxin (Fd), from species such as NADPH. Metabolic systems that use bifurcation are more efficient and confer a competitive advantage for the organisms that harbor them. Structural models are now available for two NADH-dependent ferredoxin-NADP ${ }^{+}$oxidoreductase (Nfn) complexes. These models, together with spectroscopic studies, have provided considerable insight into the catalytic process of FBEB. However, much about the mechanism and regulation of these multisubunit proteins remains unclear. Using hydrogen/deuterium exchange mass spectrometry (HDX-MS) and statistical coupling analysis (SCA), we identified specific pathways of communication within the model FBEB system, Nfn from Pyrococus furiosus, under conditions at each step of the catalytic cycle. HDX-MS revealed evidence for allosteric coupling across protein subunits upon nucleotide and ferredoxin binding. SCA uncovered a network of co-evolving residues that can provide connectivity across the complex. Together, the HDX-MS and SCA data show that protein allostery occurs across the ensemble of iron-sulfur cofactors and ligand binding sites using specific pathways that connect domains allowing them to function as dynamically coordinated units.
\end{abstract}

\section{Introduction}

NADH-dependent ferredoxin-NADP ${ }^{+}$oxidoreductase (Nfn) catalyzes the formation of reduced ferredoxin $\left(\mathrm{Fd}_{\mathrm{Red}}\right)$, from the less energetic donor, nicotinamide adenine dinucleotide phosphate $\left(\mathrm{NADP}^{+} / \mathrm{NADPH}\right)$, by coupling this reaction to the thermodynamically favorable reduction of nicotinamide adenine dinucleotide $\left(\mathrm{NAD}^{+} / \mathrm{NADH}\right)$, through a process termed flavin-based electron bifurcation (FBEB) $[1,2]$. FBEB modulates the energy metabolism within a cell by coupling the oxidation of an electron donor with an intermediate reduction potential to promote the reduction of two electron acceptors, one with a high reduction potential and one with a low potential. Doing so allows the formation of low potential, high-energy compounds, such as hydrogen gas and reduced Fd. Due to the relatively recent discovery of FBEB, the mechanism behind catalysis remains largely unresolved. However, crystal structures of the bifurcating enzyme Nfn were successfully obtained from Thermotoga maritima (Tm) [3] and Pyrococcus furiousus (Pf) [4] which enhances the ability to study the FBEB mechanism using this enzyme.

$\mathrm{Nfn}$ is a heterodimeric protein comprising a small (Nfn-S) and large

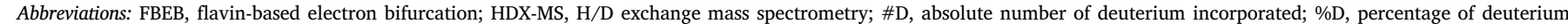

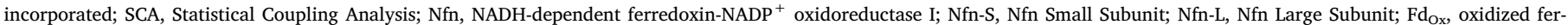

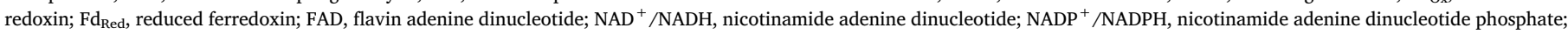
Tm, Thermotoga maritima; Pf, Pyrococcus furiosus 

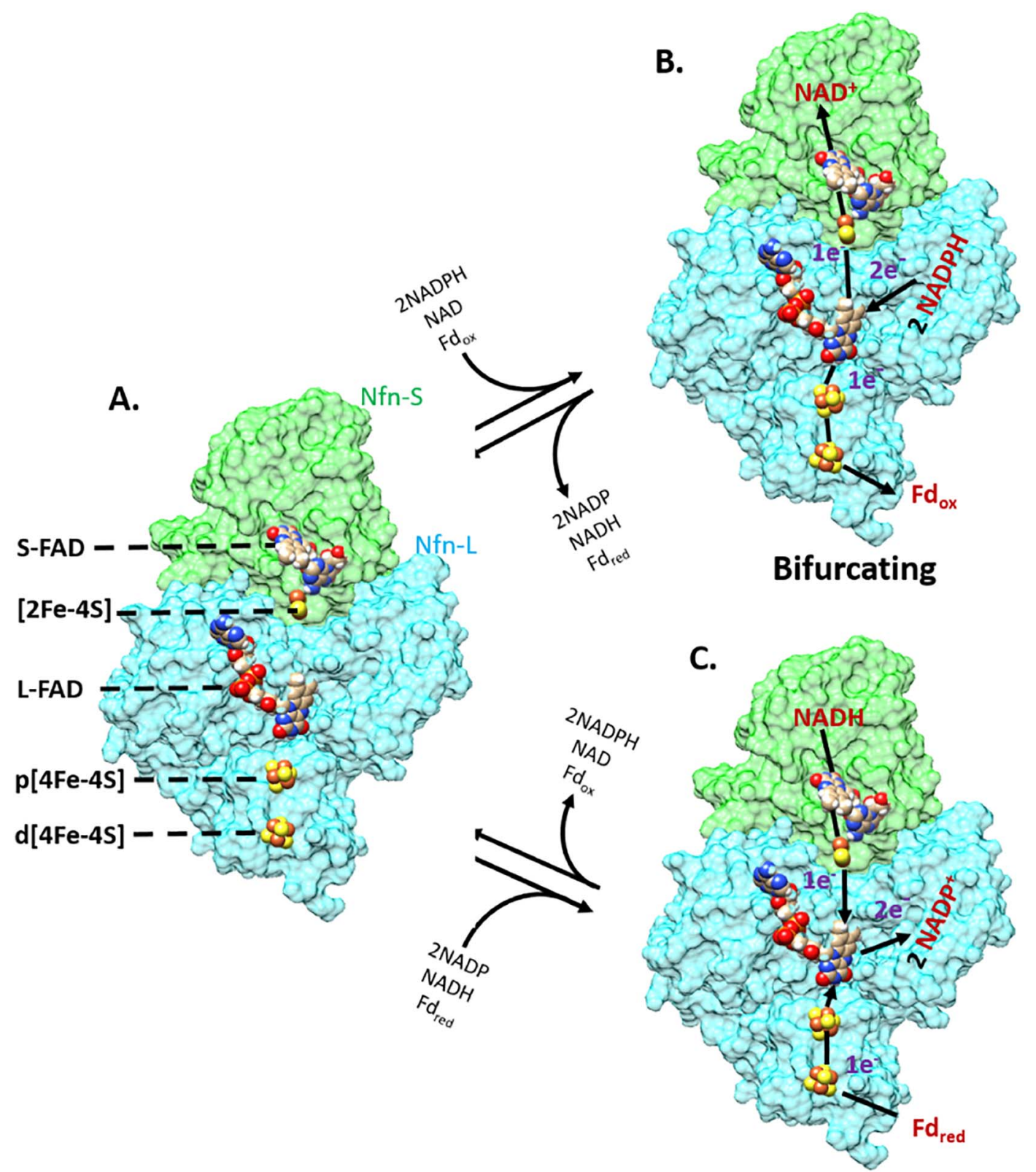

\section{Confurcating}

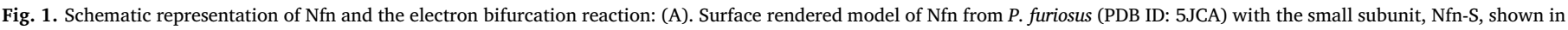

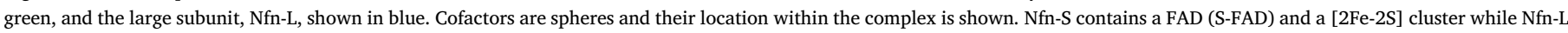

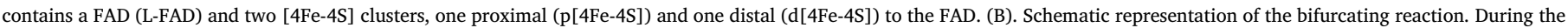

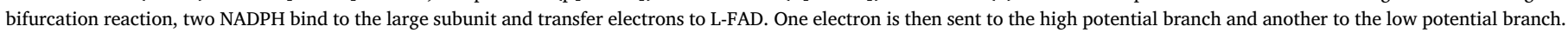

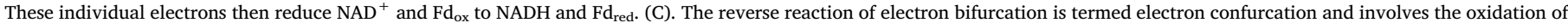

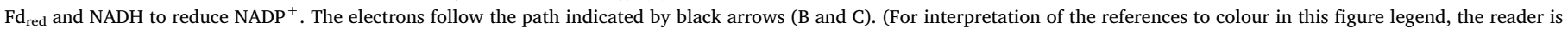
referred to the web version of this article.)

(Nfn-L) subunit (Fig. 1A) [4,5]. Each of these subunits have cofactors involved in the bifurcation reaction, including two flavin adenine dinucleotides (FAD), one in each subunit, a proximal [4Fe-4S] cluster ( $\mathrm{p}$ [4Fe-4S]) in Nfn-L, a distal [4Fe-4S] cluster (d[4Fe-4S]) in Nfn-L, and one $[2 \mathrm{Fe}-2 \mathrm{~S}]$ cluster in Nfn-S. These cofactors allow Nfn to catalyze the formation of reduced Fd, from NADPH and $\mathrm{NAD}^{+}$(Eq. (1), Fig. 1B). The enzyme can also catalyze the reverse confurcating reaction to form NADPH by oxidizing Fd and NADH (Fig. 1C); this is thought to be the physiological role of $\mathrm{Nfn}$.

$2 \mathrm{NADPH}+\mathrm{NAD}^{+}+\mathrm{Fd}_{\mathrm{Ox}} \rightleftharpoons 2 \mathrm{NADP}^{+}+\mathrm{NADH}+\mathrm{Fd}_{\mathrm{Red}}^{2-}+\mathrm{H}^{+}$

Studies of Nfn purified from $P f$ and $T m$ have revealed that NADH/
$\mathrm{NAD}^{+}$binds to Nfn-S, and NADPH/NADP ${ }^{+}$binds to Nfn-L, and Fd is predicted to bind to Nfn-L near the distal [4Fe-4S] cluster $[4,6]$. Recent studies have revealed that the bifurcation reaction proceeds by the formation of a highly unstable flavin anionic semiquinone (ASQ) that forms through the oxidation of NADPH, and this ASQ has sufficient energy to reduce Fd [4]. A key feature of bifurcating enzymes, specifically Nfn from $P f$, that remains unknown is how energy is conserved during the exergonic reduction reaction, how this energy is then used to drive the endergonic reaction, and whether gating of electrons might be involved in either of these processes. Studying the effects of substrate binding on the structure and dynamics of Nfn may provide critical new information regarding the role of protein structure in modulating FBEB. 
While previous studies have described the structure and catalytic properties associated with Nfn $[7,8]$, the finer mechanistic details of FBEB in this enzyme largely remain unknown. In a recent study of $T m$ Nfn using H/D exchange mass spectrometry (HDX-MS), it was shown that binding of the nucleotide NADPH/NADP ${ }^{+}$to Nfn-L resulted in a subtle conformational change near the proposed Fd binding site [3], which suggested the possibility of long distance communication between the nucleotide and Fd binding sites. This observation was made possible through the application of the powerful technique, HDX-MS, that tracks exchange of amide hydrogens in the protein backbone with water via a base-catalyzed reaction [9]. By replacing water $\left(\mathrm{H}_{2} \mathrm{O}\right)$ with deuterated water $\left(\mathrm{D}_{2} \mathrm{O}\right)$, the amide hydrogens that exchange will be replaced with deuterium which can be tracked using mass spectrometry. The tertiary and quaternary structure of a protein, as well as the stability of the hydrogen bonds within the secondary structural elements, influence the rate by which H/D exchange occurs $[10,11]$. This allows HDX-MS to reveal substrate/ligand binding sites, points of contact between subunits in a complex, and changes in dynamics even in peptides that participate in allosteric communication within a single protein, or an entire complex $[12,13]$.

Conformational changes caused by ligand binding are increasingly being recognized as an intrinsic feature involved in the functionality of many proteins [14]. The ability of ligands to induce conformational changes at a site remote from the site of binding has been termed allostery [15]. However, the pathways of allosteric communication in a protein complex, or even a single protein are rarely known. One method that has proven useful for mapping allosteric pathways of connectivity and can provide mechanistic insights on protein regulation is Statistical Coupling Analysis (SCA). SCA is a method that identifies co-varying (coevolving) suites of amino acid residues that exhibit evidence for energetic connectivity, which in turn has been suggested to be evidence for possible allosteric communication within the protein $[15,16,17]$. SCA quantifies how changes in the distribution of amino acids at one position influence changes in the amino acid distribution at another position. Energetic connectivity is then computed using a curated sequence alignment of homologs, within the context of a combined statistical and protein structural framework.

To investigate the potential role of allosteric communication in FBEB by $P f$ Nfn, we used SCA to identify potential pathways of communication within the enzyme complex and HDX-MS to study changes in the stability and dynamics of the protein complex when different substrates are present. Analyses were conducted on enzymes in the presence and absence of nucleotides and $\mathrm{Fd}_{\mathrm{Ox}}$. Our HDX-MS results reveal that ligand binding sites display protection from exchange when substrates are bound to a distant site, suggesting allosteric communication. SCA analyses identified 137 residues in Nfn that form an allosteric communication pathway, providing strong evidence supporting the role of ligand induced allosteric communication during FBEB. Additionally, changes in H/D suggests a role for protein dynamics and communication pathways in tuning electron transfer by influencing the protein microenvironment around the cofactors.

\section{Materials and methods}

\subsection{Protein purification}

The $P f$ Nfn complex (Nfn-SL) and Fd were purified from $P$. furiosus, as previously described [4].

\subsection{Native mass spectrometry}

Non-covalent mass spectrometry experiments were conducted on a SYNAPT G2-Si instrument (Waters) in a similar fashion as described previously [18]. Briefly, the Nfn complex sample was buffer exchanged to $200 \mathrm{mM}$ ammonium acetate and $\mathrm{pH} 7$ (Sigma) using $3 \mathrm{kDa}$ molecular weight cutoff spin filters (Pall Corporation). Nfn was then infused from in-house prepared gold-coated borosilicate glass capillaries to the electrospray source at a protein concentration of $5 \mu \mathrm{M}$ and a rate of roughly $90 \mathrm{~nL} / \mathrm{min}$. The instrument was tuned to enhance performance in the high mass-to-charge range. Settings were as follows: Source temperature $30^{\circ} \mathrm{C}$, capillary voltage of $1.7 \mathrm{kV}$, trap bias voltage of $16 \mathrm{~V}$, and argon flow in the collision cell (trap) of $7 \mathrm{~mL} / \mathrm{min}$. Transfer collision energy was held at $10 \mathrm{~V}$ while trap energy varied between 10 and 200 V. Data analysis was performed in the MassLynx software version 4.1 (Waters).

\subsection{HDX-MS analysis}

Nfn was subjected to HDX-MS analysis under eight conditions: 1) aspurified, 2) NADH bound, 3) $\mathrm{NAD}^{+}$bound, 4) $\mathrm{NADP}^{+}$bound, 5) $\mathrm{NADPH}$ bound, 6) $\mathrm{NADH}+\mathrm{NADP}^{+}$bound, 7) $\mathrm{NADPH}+\mathrm{NAD}^{+}$ bound, and 8) $\mathrm{Fd}_{\mathrm{Ox}}$ bound. Pyridine nucleotides were incubated with $P f$ $\mathrm{Nfn}$ at a final concentration of $1 \mathrm{mM}$. Nfn:Fd $\mathrm{Ox}_{\mathrm{X}}$ complex was formed by first making a 1:1 $\mathrm{M}$ ratio of $\mathrm{Nfn}$ to $\mathrm{Fd}_{\mathrm{Ox}}$ using $10 \mu \mathrm{L}$ of $\mathrm{Nfn}(16.58 \mathrm{mg} /$ $\mathrm{mL}$ ) followed by incubation under anoxic conditions for $1 \mathrm{~h}$ at room temperature $\left(\sim 21{ }^{\circ} \mathrm{C}\right)$ before initiating the reaction. The exchange reaction was initiated by a 10 -fold dilution of $\mathrm{Nfn}$ with reaction buffer at $60{ }^{\circ} \mathrm{C}$. This temperature was chosen due to temperature limitations of the equipment available. Reaction buffer contained $100 \mathrm{mM}$ Tris- $\mathrm{HCl}$ and $150 \mathrm{mM} \mathrm{NaCl}$ in $\mathrm{D}_{2} \mathrm{O}$ (pD 7.0) with $1 \mathrm{mM}$ of pyridine nucleotides $\left(\mathrm{NAD}^{+}, \mathrm{NADH}, \mathrm{NADP}^{+}, \mathrm{NADPH}, \mathrm{NADPH}+\mathrm{NAD}^{+}\right.$, or $\mathrm{NADH}$ $+\mathrm{NADP}^{+}$). Samples were removed and quenched to stop exchange after $1 \mathrm{~min} ., 3 \mathrm{~min} ., 15 \mathrm{~min} ., 60 \mathrm{~min} ., 3 \mathrm{~h}$, and $24 \mathrm{~h}$. At each time point, a $10 \mu \mathrm{L}$ subsample was withdrawn from each reaction vial and placed into quenching/digestion solution containing $1 \%$ formic acid (FA, Sigma) and porcine pepsin (Sigma, $0.2 \mathrm{mg} / \mathrm{mL}$ final concentration) on ice. After a two min. incubation, the reaction mixture was frozen in liquid $\mathrm{N}_{2}$ and stored at $-80{ }^{\circ} \mathrm{C}$ until it was subjected to liquid chromatography-mass spectrometry (LC-MS) analysis.

LC-MS analysis of Nfn peptide fragments was completed on a 1290 UPLC series chromatography stack (Agilent Technologies) coupled directly to a 6538 UHD Accurate-Mass Q-TOF LC/MS mass spectrometer (Agilent Technologies). Before electrospray-time of flight (ESI-TOF) analysis, peptides were separated on a reverse phase (RP) column (Phenomenex Onyx Monolithic C18 column, $100 \times 2 \mathrm{~mm}$ ) at $1{ }^{\circ} \mathrm{C}$ using a flow rate of $500 \mu \mathrm{L} / \mathrm{min}$. Under the following conditions: 0.0-1.0 min., 5\% B; 1.0-9.0 min., 5-45\% B; 9.1-9.8 min., 95\% B; 9.8-9.9 min., 5\% B. Solvent A contained $0.1 \%$ FA in water (ThermoFisher) while solvent B contained $0.1 \%$ FA in acetonitrile (ACN, ThermoFisher). Data was acquired at $2 \mathrm{~Hz}$ over a scan range $50-1700 \mathrm{~m} / \mathrm{z}$ in positive mode. Electrospray settings were: nebulizer at 3.7 bar, drying gas at $8.0 \mathrm{~L} / \mathrm{min}$., drying temperature at $350{ }^{\circ} \mathrm{C}$, and capillary voltage at $3.5 \mathrm{kV}$. Data dependent MS/MS, specifying a selection window of $4 \mathrm{~m} / z$, was used to generate peptide sequence tags. Data processing was carried out in Agilent MassHunter Qualitative Analysis version 6.0. Peptide identification was performed using the peptide analysis worksheet (PAWs, ProteoMetrics LLC.). Deuterium uptake was determined by monitoring shifts of the centroid peptide isotopic distribution by using the program HDExaminer (Sierra Analytics Inc.). Measured values were then used to generate uptake curves to compare relative deuterium exchange in all conditions tested.

\subsection{HDX-MS clustering}

The percent deuterium (\%D) incorporated at a given time point was calculated by dividing the number of deuterium atoms incorporated (\#D) by the number of deuterium atoms incorporated after $24 \mathrm{~h}$. The percent difference between the deuterium uptake in the as-purified condition and the percent deuterium incorporated during experimental manipulation was calculated as published by Chalmers et al. [19]. Briefly, the $\% \mathrm{D}$ of the as-purified condition (no substrate bound) was then subtracted from the $\% \mathrm{D}$ of the nucleotide bound and $\mathrm{Fd}_{\mathrm{Ox}}$ bound 


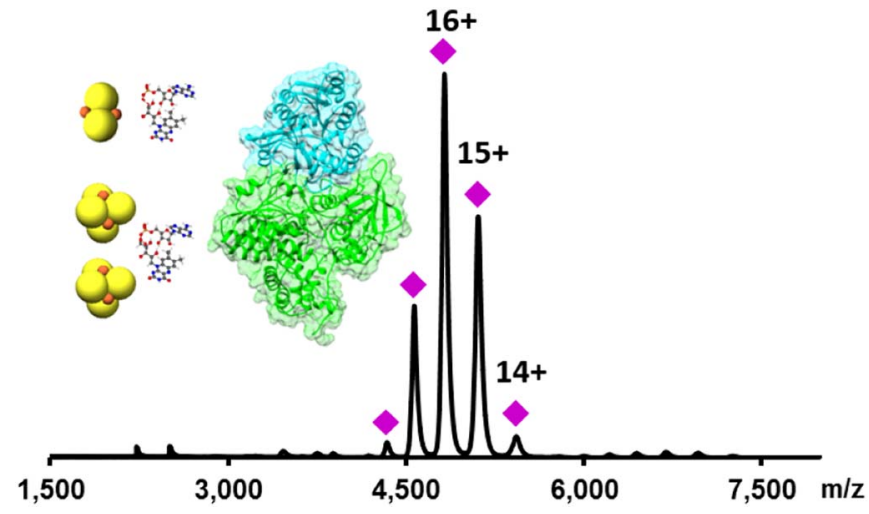

Fig. 2. Native mass spectrum of Pf Nfn complex (as-purified) in the gas phase. The intact complex (magenta diamonds represent charge envelope centered around $16+$ charge) contains two subunits (Nfn-S, blue and Nfn-L, green), two FAD, two [4Fe-4S] clusters and one [2Fe-2S] cluster. The mass of the complex based on deconvoluted signal is $86,407 \mathrm{Da}$ (calculated $\mathrm{MW}=86,425 \mathrm{Da}$ ). (For interpretation of the references to colour in this figure legend, the reader is referred to the web version of this article.)

conditions. The difference was then plotted for peptides with sufficient deuterium incorporation at the early (60 s.), middle ( $900 \mathrm{s.}$ ), and late $(10,800 \mathrm{~s}$.) time points. A hierarchical clustering script in $\mathrm{R}$ ( $\mathrm{R}$ version 3.0.0) was used to determine the number of trends present within the deuterium uptake profiles based on the slope of the treadline. For instance, linear treadlines with a positive slope were clustered together, whereas profiles with a polynomial treadline were clustered depending on if the treadline continually increased, continually decreased, if the parabola formed is positive or negative, or if there is no change throughout the time course (Supp. Figs. 1-7). The clustered profiles were then implemented in a network analysis (described below).

\subsection{Generation of Nfn and related paralog database}

A database of Nfn-S and Nfn-L homologs, compiled as part of our previous study [5], was used here. Briefly, the database was constructed by subjecting Nfn-S and Nfn-L from Pf (AAD36706 and AAD36707, respectively) to separate BLASTp queries against the National Center for Biotechnology Information (NCBI) complete genome database ( $n=4586$ genomes as of March 2016) specifying thresholds of $>30 \%$ amino acid identity and $>60 \%$ coverage. Only Nfn-S and Nfn-L sequences that were co-localized in the genome were retained for further analysis. Compiled Nfn-S and Nfn-L homologs were aligned with Clustal Omega specifying default settings and were then manually screened to filter out non-Nfn sequences using residues previously suggested to be essential to protein function, as outlined in Demmer et al. $[6,20]$. These steps resulted in a curated database of 467 Nfn-SL homologous sequences.

\subsection{Statistical coupling analysis (SCA)}

Aligned homologous sequences of both Nfn-S and Nfn-L $(n=467)$ were concatenated using a custom python script (python version 2.7). The concatenated sequences were then curated to include only positions that contained a maximum gap frequency of $20 \%$, resulting in a total of 750 positions (out of 1955 positions including gaps). The curated Nfn-SL sequences along with the $P f$ Nfn structure (PDB: 5JFC) were subjected to SCA analysis in MATLAB (MATLAB version 9.1) as previously described in Halabi et al. 2009 [17]. Chimera (version 3.2.0) was used to map and visualize the spatial location of positions that exhibited evidence for co-variance (co-evolution) in the $P f \mathrm{Nfn}$ structure [21].

\subsection{Network analysis}

The matrix obtained by clustering the HDX-MS data was subjected to Pearson correlation using the correlation package in Cytoscape (version 3.2.0) [22]. The network was visualized with the force directed organic layout [23]. Each unique peptide was denoted as a node and the edges between nodes represent the degree of correlation between HDX exchange between a given set of peptides (nodes). The network was further analyzed using the Network Analyzer plugin in Cytoscape to map the correlation information onto the network [24]. Network nodes were colored based on results from SCA analysis.

\section{Results}

\subsection{Evidence of long distance communication in $P f N f n$}

To investigate the role of structural dynamics in the mechanism of FBEB in Nfn, we applied HDX-MS to $P f$ Nfn poised at different steps in the catalytic cycle. Individual steps were simulated by adding pyridine nucleotides and $\mathrm{Fd}_{\mathrm{Ox}}$ to oxidized Nfn in seven different combinations. Deuterium uptake over time was then compared in the as-purified Nfn enzyme with that observed when provided with pyridine nucleotides or $\mathrm{Fd}_{\mathrm{Ox}}$. Before beginning experiments, the as-purified Nfn complex from $P f$ was analyzed by native MS to confirm its composition. Native MS showed that $P f \mathrm{Nfn}$ contains two FAD molecules, two [4Fe-4S] clusters, and one [2Fe-2S] cluster (Fig. 2). Based on native MS data collected at low collision energy, cofactor occupancy is estimated to be $100 \%$. This analysis also provided assurance that pyridine nucleotides and $\mathrm{Fd}_{\mathrm{Ox}}$ did not co-purify with the Nfn enzyme. A pepsin digestion of Nfn produced $>200$ peptides that could each be uniquely mapped to the amino acid sequence of $P f$ Nfn. From these 200 peptides, 97 and 125 were used as input to generate deuterium uptake curves for Nfn-S and $\mathrm{Nfn}-\mathrm{L}$, respectively. Sequence coverage was $100 \%$ for Nfn-S and $94 \%$ for Nfn-L for the peptides chosen (Supp. Fig. 8). These peptides were used to map specific regions of deuterium incorporation during HDXMS.

Of the identified peptides, a subset from each subunit displayed significantly different levels of deuterium incorporation dependent on nucleotide binding. We reasoned that peptides displaying changes in dynamic behavior upon substrate binding are important in the mechanism underpinning FBEB and catalytic activity. Also, since the deuterium uptake in a number of peptides did not change under any of the conditions tested, we are confident that the observed changes are not an artifact of ligand binding [25]. Heat maps of the selected peptides show a global view of exchange in Nfn-S (Supp. Fig. 9) and Nfn-L (Supp. Fig. 10) as a function of incubation time. The heat maps show that the as-purified state of Nfn had the most deuterium incorporation while the NADPH $+\mathrm{NAD}^{+}$bound form had the lowest deuterium incorporation. Uptake curves for each of the peptides provide a local view of the deuterium incorporation in each condition during the course of the reaction (Supp. Figs. 11 and 12).

To investigate the possible role of protein dynamics in the mechanism of FBEB, we focused additional analyses on peptides in close proximity to the cofactors and nucleotide binding sites. Six key peptides (two in Nfn-S and four in Nfn-L) were selected for a detailed analysis. All peptide residue numbers are relative to Nfn from $P f$. The two peptides from Nfn-S (residues 131-139 and 210-226) are involved in $\mathrm{NADH} / \mathrm{NAD}^{+}$binding and S-FAD/[2Fe-2S] cluster coordination, respectively $[4,6]$. The other four peptides are located in Nfn-L; residues 43-64 ([4Fe-4S] cluster binding/near putative Fd docking site), 70-85 (control region), 245-262 (L-FAD binding domain), and 370-392 (stabilizing loop upon NADPH/NADP ${ }^{+}$binding).

To visualize changes in the exchange profile of Nfn upon nucleotide and Fd binding, we created a histogram plot comparing HDX data for the $3 \mathrm{~h}$ time point. Nfn-S residues $131-139$, involved in NADH/NAD ${ }^{+}$ binding, showed a $30 \%$ decrease in deuterium incorporation when 


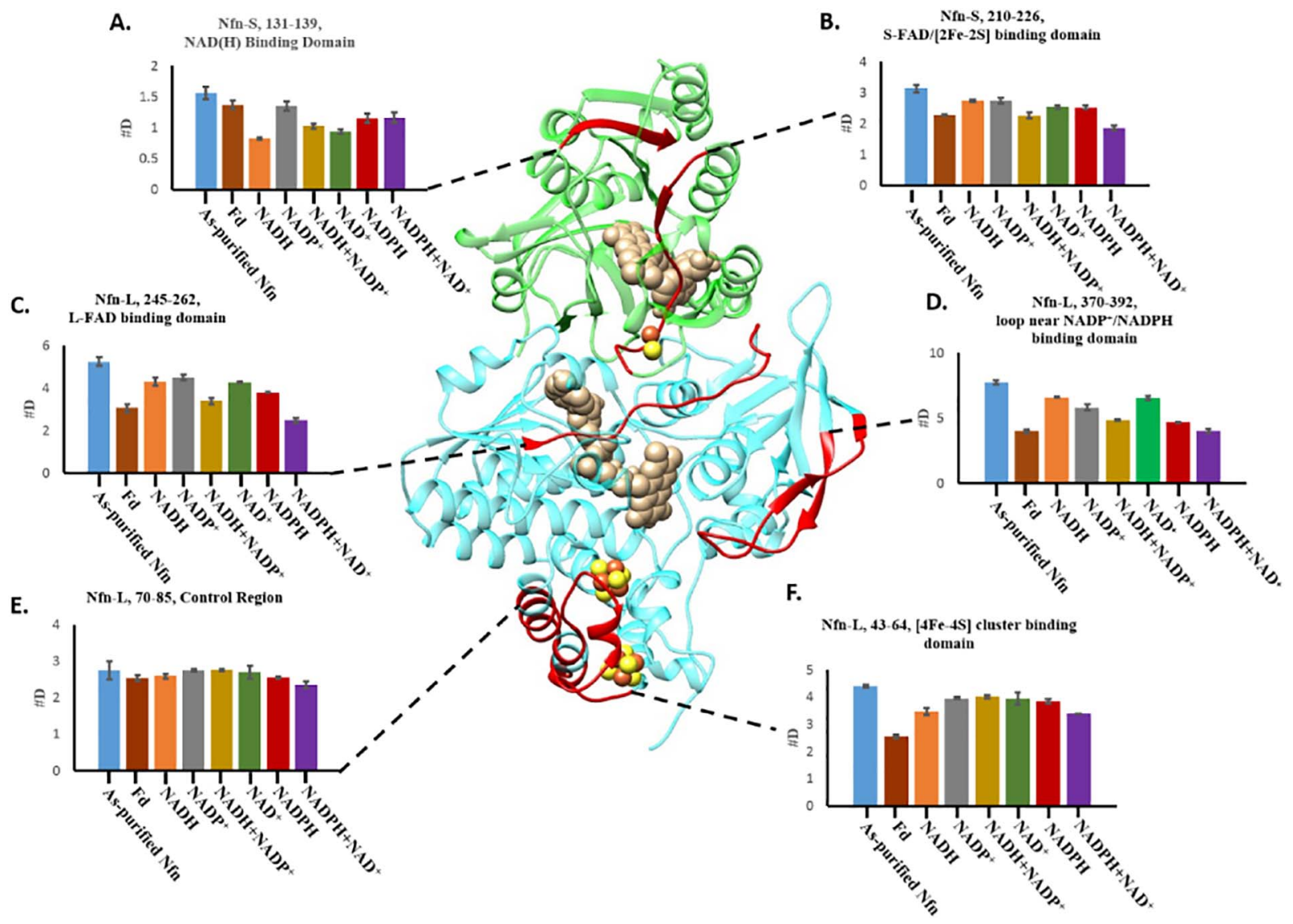

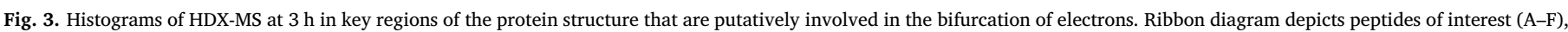

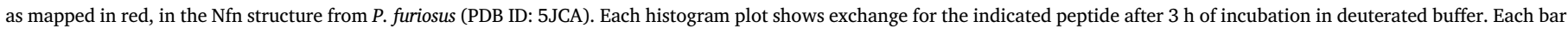

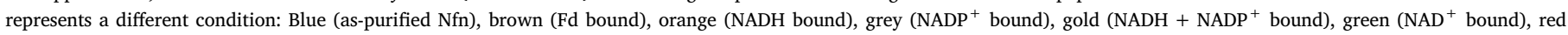

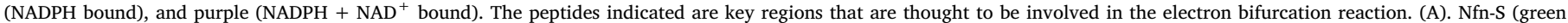

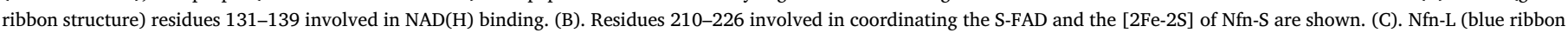

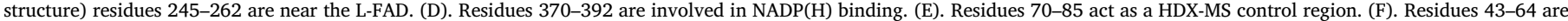

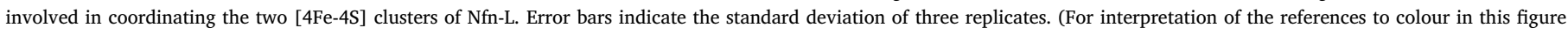
legend, the reader is referred to the web version of this article.)

$\mathrm{NADH}$ or $\mathrm{NAD}^{+}$are bound, when compared to the as-purified condition (Fig. 3A). When the nucleotides $\mathrm{NADP}^{+}$or NADPH bind to Nfn-L, a decrease in deuterium incorporation of 10 and $20 \%$, respectively, occurs at residues 131-139 of Nfn-S. This suggests that the binding of $\mathrm{NADP}^{+}$or NADPH to Nfn-L induces a change in the structure or stability of Nfn-S. When Fd is bound to Nfn-L, residues 131-139 of Nfn-S incorporate $10 \%$ less deuterium than the as-purified condition. This indicates a change in the secondary structure stability in this region. The remaining conditions tested, $\mathrm{NADH}+\mathrm{NADP}^{+}$, and $\mathrm{NADPH}$ $+\mathrm{NAD}^{+}$, both exchange less deuterium than the as-purified condition, with the NADH $+\mathrm{NADP}^{+}$condition exhibiting less exchange than the $\mathrm{NADPH}+\mathrm{NAD}^{+}$condition. Residues 210-226 in Nfn-S exhibited a $20 \%$ and $<10 \%$ decrease in deuterium incorporation when NADH $+\mathrm{NADP}^{+}$are both bound or when the nucleotides are individually bound to Nfn, respectively (Fig. 3B). Similarly, when NADPH $+\mathrm{NAD}^{+}$ are both bound, there is a $30 \%$ decrease in exchange at residues 210-226 in Nfn-S, indicating that this condition has the greatest effect on the structure or stability of this peptide.

Of the four peptides that were closely examined in Nfn-L, residues 70-85 exhibited small changes in deuterium incorporation in all conditions (Fig. 3E) and thus can be considered as one of the control regions for the HDX experiments. Other control regions include residues 128-141 of Nfn-S, and residues 302-323 of Nfn-L (Supp. Fig. 11E and Supp. Fig. 12M). The remaining three peptides in Nfn-L (43-64, 245-262, and 370-392) displayed evidence of synergistic exchange dynamics during nucleotide binding (Fig. 3C, D, and F). Nfn-L residues
245-262, which are in close proximity to the L-FAD, exhibit high amounts of exchange in the as-purified, NADH bound, NADP ${ }^{+}$bound, $\mathrm{NAD}^{+}$bound, and NADPH bound conditions. Among these conditions the NADH and $\mathrm{NADP}^{+}$conditions exchanged $15 \%$ and $10 \%$ less deuterium relative to the as-purified condition, respectively, whereas $\mathrm{NAD}^{+}$and NADPH bound exchanged $20 \%$ and $25 \%$ less deuterium, respectively (Fig. 3C). The least amount of exchange was observed in this peptide when $\mathrm{Fd}_{\mathrm{Ox}}, \mathrm{NADH}+\mathrm{NADP}^{+}$, or $\mathrm{NADPH}+\mathrm{NAD}^{+}$, are bound. Relative to as-purified Nfn, a 45\%, 30\%, and 35\% decrease in deuterium exchange was observed in the NADPH $+\mathrm{NAD}^{+}, \mathrm{NADH}$ $+\mathrm{NADP}^{+}$, and Fd bound states, respectively (Fig. 3C).

Residues 370-392 of Nfn-L are involved in a loop that becomes less flexible, and therefore less accessible to exchange, upon NADPH/ $\mathrm{NADP}^{+}$binding because of the interactions formed between the loop and the $\mathrm{NADPH} / \mathrm{NADP}^{+}$binding pocket [4]. Importantly, $\mathrm{Fd}_{\mathrm{Ox}}$ and $\mathrm{NADPH}+\mathrm{NAD}^{+}$binding results in a decrease of $40 \%$ in the amount of deuterium incorporated in this peptide. This indicates that residues 370-392 are the least accessible to exchange under these two conditions. Additionally when $\mathrm{NADP}^{+}, \mathrm{NADPH}$, and $\mathrm{NADH}+\mathrm{NADP}^{+}$are bound to Nfn, a decrease in deuterium incorporation $(20 \%, 30 \%$, and $30 \%$, respectively) is observed at residues $370-392$, albeit not as much of a decrease when compared to $\mathrm{Fd}_{\mathrm{Ox}}$, or $\mathrm{NADPH}+\mathrm{NAD}^{+}$bound conditions ( $40 \%$ decrease in both conditions). The remaining two conditions, with $\mathrm{NADH}$ or $\mathrm{NAD}^{+}$bound, display a $15 \%$ decrease in exchange at residues 370-392 relative to the as-purified condition. This is the opposite of the results for Nfn-S where a decrease of $10 \%$ and 
$20 \%$ is observed in residues $131-139$ when $\mathrm{NADP}^{+}$or $\mathrm{NADPH}$ are bound to Nfn-L.

The fourth peptide of interest in Nfn-L (residues 43-64) is responsible for coordinating both [4Fe-4S] clusters, and is believed to be near the site of Fd binding to the large subunit [4]. Fd is expected to bind near the distal FeS cluster on the large subunit, close to the 43-64 region $[3,4]$. It was observed that $\mathrm{Fd}_{\mathrm{Ox}}$ binding to $\mathrm{Nfn}$ caused increased protection from exchange in residues 43-64 of Nfn-L. This observation is consistent with previous studies of protein-protein and protein-ligand interactions, in which a large decrease in exchange indicates a site of possible interaction between the two subjects of interest $[26,27]$.

\subsection{SCA analysis of Nfn}

Data generated from HDX-MS revealed evidence of long distance communication within the complex. To elucidate specific details about potential pathways of communication across Nfn, we utilized SCA. SCA can reveal energetically linked, or co-evolving, amino acid residues. A structure-based sequence alignment was constructed of 467 Nfn-like proteins. From this, a total of 137 co-evolving positions were identified of which 52 positions were in Nfn-S and 85 were in Nfn-L (Supp. Table 1). Of the 52 co-evolving residues identified in Nfn-S, 18 residues were within $10 \AA$ of the S-FAD and another two were within $10 \AA$ the [2Fe-2S] cluster in Nfn-S. Of these 20 residues, six that were in close proximity to S-FAD were highly conserved (i.e., $>80 \%$ ) and one residue near the $[2 \mathrm{Fe}-2 \mathrm{~S}]$ cluster was also highly conserved (Supp. Table 1). In Nfn-L, 16 of the 85 residues were within $10 \AA$ Af the L-FAD, two residues were located within $10 \AA$ of the proximal [4Fe-4S] cluster, and six residues were within $10 \AA$ of the distal [4Fe-4S] cluster. Of the 16 residues that were near the L-FAD, two were highly conserved (Supp. Table 1).

Residues that were $100 \%$ conserved (a total of 50 residues) were not included in the SCA due to the lack of variation among homologous proteins used in the multiple sequence alignment. Twelve of the $100 \%$ conserved residues were identified in Nfn-S and 38 were identified in Nfn-L (Supp. Table 2). Of the 12 identified in Nfn-S, five are within $10 \AA$ of the [2Fe-2S] cluster and six are within $10 \AA$ of the S-FAD. In Nfn-L, five $100 \%$ conserved residues were located near the proximal [4Fe-4S] cluster, five were located near the distal [4Fe-4S] cluster, and 21 were located near the L-FAD.

To visualize and identify a putative communication pathway in Nfn, the combination of residues that were $100 \%$ conserved in our sequence dataset and those that exhibited evidence for co-evolution from SCA were mapped onto the $P f \mathrm{Nfn}$ structure. A total of 14 residues were identified that were within $6 \AA$ from one another or from the cofactors involved in electron transfer (Fig. 4); all of the residues identified were $>70 \%$ conserved. Of the 14 residues, six were identified in Nfn-S, connecting the $\mathrm{NAD}(\mathrm{H})$ binding site to L-FAD. Three of the six residues in Nfn-S are present in peptides identified via HDX-MS analysis: Glycine at position 138 within the 131-139 region peptide that interacts with $\mathrm{NADH} / \mathrm{NAD}^{+}$, G112 in peptide $95-113$ that interacts with the S-FAD, and G226 that interacts with the [2Fe-2S] cluster. Likewise, eight of the 14 residues were identified in Nfn-L that connect L-FAD to the NADPH/ $\mathrm{NADP}^{+}$and Fd binding sites. Two of these residues were detected by HDX-MS as being involved in deuterium exchange: Proline at position 252 (P252) in peptide 242-262 that interacts with L-FAD and cysteine at position 45 (C45) in peptide 43-64 that interacts with the distal [4Fe4S] cluster and Fd.

\subsection{Network analysis of HDX-MS and SCA data}

Protein function is an emergent property arising from the integration of functional units. We reasoned that the biophysical properties and evolutionary fingerprint could be combined to elucidate underlying modules that give rise to protein functionality. Peptides were clustered based on the percent difference in deuterium uptake between the as- purified and different pyridine nucleotide/ $/ \mathrm{Fd}_{\mathrm{Ox}}$ bound conditions. Thirty six of the mapped peptides from Nfn were used. These peptides all had significant deuterium incorporation $(\# \mathrm{D}>1)$ and were proximal to key regions of the protein. We also selected several peptides in which little to no difference in exchange was observed between the tested conditions. The resulting matrix was analyzed by Pearson correlation and converted to a network map for visualization. This network was then overlayed with the SCA data which identified evidence for coevolving residues within the designated peptides. Two networks were generated by separating the bifurcating and confurcating reactions in order to reveal pathways of connectivity (Fig. 5A and Supp. Fig. 13A). The bifurcating and confurcating reactions were separated based on the pyridine nucleotides needed to perform the reaction in a specified direction (see Eq. (1)). For instance, the bifurcating reaction uses $\mathrm{NAD}^{+}$, $\mathrm{NADPH}$, and $\mathrm{Fd}_{\mathrm{Ox}}$, whereas the confurcating reaction uses NADH, $\mathrm{NADP}^{+}$, and $\mathrm{Fd}_{\text {Red }}$. The HDX conditions involving one or both of the nucleotides that are required to drive reaction directionality were grouped together to create the network plots.

The bifurcation network revealed two distinct clusters of peptides based on HDX (Fig. 5A). One cluster corresponds to peptides that exhibit high levels of HDX exchange under conditions (NADPH, $\mathrm{NAD}^{+}$, $\mathrm{NADPH}+\mathrm{NAD}^{+}$, and $\mathrm{Fd}_{\mathrm{Ox}}$ bound) that promote bifurcation, while a second corresponds to peptides that exhibit high levels of correlation under conditions $\left(\mathrm{NADP}^{+}, \mathrm{NADH}\right.$, and $\mathrm{NADH}+\mathrm{NADP}^{+}$bound) that promote confurcation (Supp. Fig. 13). In each of these conditions, peptides (i.e., network nodes) that contained at least one residue that exhibited evidence for co-evolution with other residues within the protein are highlighted in red whereas network nodes that did not exhibit evidence for co-evolution are highlighted in green. Peptides involved in the bifurcation direction are separated into the red or purple clusters and mapped onto the Pf Nfn structure (Fig. 5B and C, respectively). Mapping of the peptides that comprise the red cluster on the $P f$ Nfn structure reveals that they are heavily centered around the cofactors of Nfn-S and Nfn-L. In contrast, mapping of the peptides that comprise the purple cluster on the $P f \mathrm{Nfn}$ structure reveals peptides that are more spread across the enzyme complex, including residues near the cofactors and the $\mathrm{NADH} / \mathrm{NAD}^{+}$binding site.

\section{Discussion}

The use of FBEB to improve metabolic efficiency in anaerobic organisms is of great interest because of the advantage provided in energy poor environments and potential application for biotechnology $[1,4]$. The key step in FBEB is the transfer of electrons from a molecule of medium reduction potential to one of lower potential (thermodynamically unfavorable) by coupling this with a transfer to a high potential acceptor (thermodynamically favorable). Our interest is in understanding the mechanism behind the splitting of electron pairs and how enzymes ensure that both electrons do not travel down the energetically favorable pathway. Current hypotheses include a "gatingstep" in which a change in the reduction potential or distance between cofactors is involved. Two recent studies that used HDX to investigate $\mathrm{Nfn}$ homologs identified evidence in support of a role for conformational change during FBEB $[4,6]$. Here we have significantly deepened our understanding of this mechanism using native mass spectrometry, HDX, in combination with SCA of $P f \mathrm{Nfn}$ at each step of the catalytic cycle.

The first step in our analysis was to establish the subunit and cofactor stoichiometry of purified Nfn. Using native MS, it was determined that Nfn purified from $P f$ contained the predicted cofactors (two [4Fe-4S] clusters, one [2Fe-2S], and two FADs) for the holo-enzyme complex (Fig. 2). This is the first native MS of an oxygen sensitive multisubunit protein complex and opens the door for investigating other systems under strictly anaerobic conditions using this powerful technique.

Many proteins utilize long distance communication between 

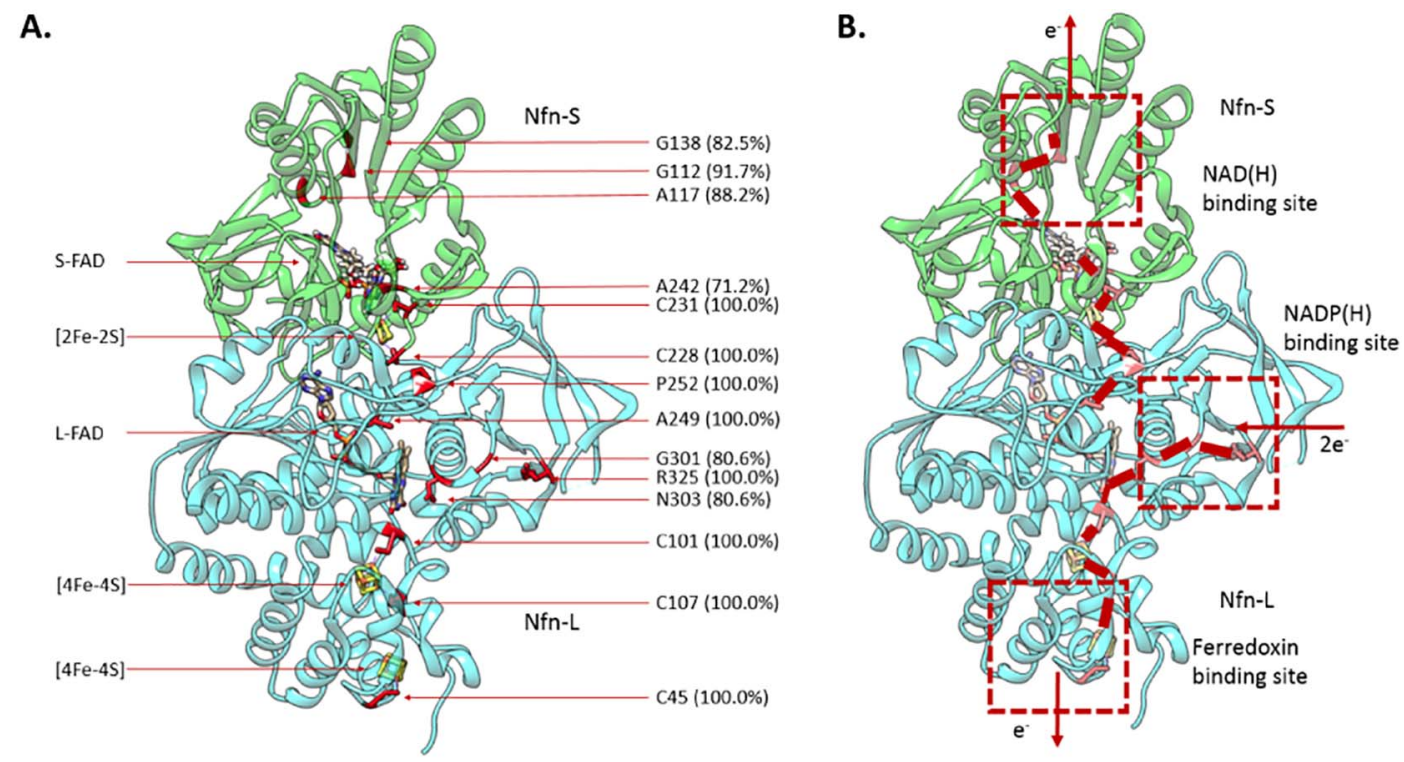

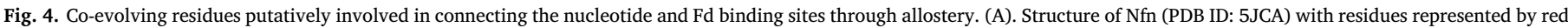

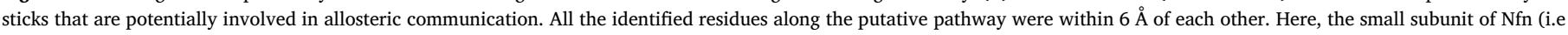

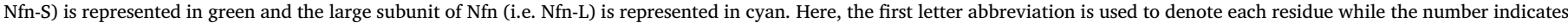

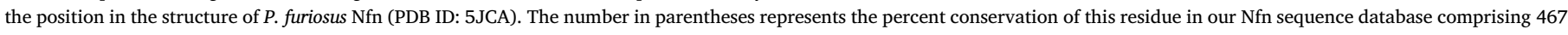

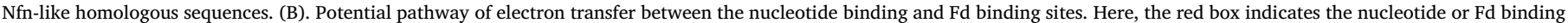

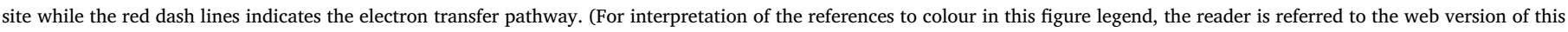
article.)
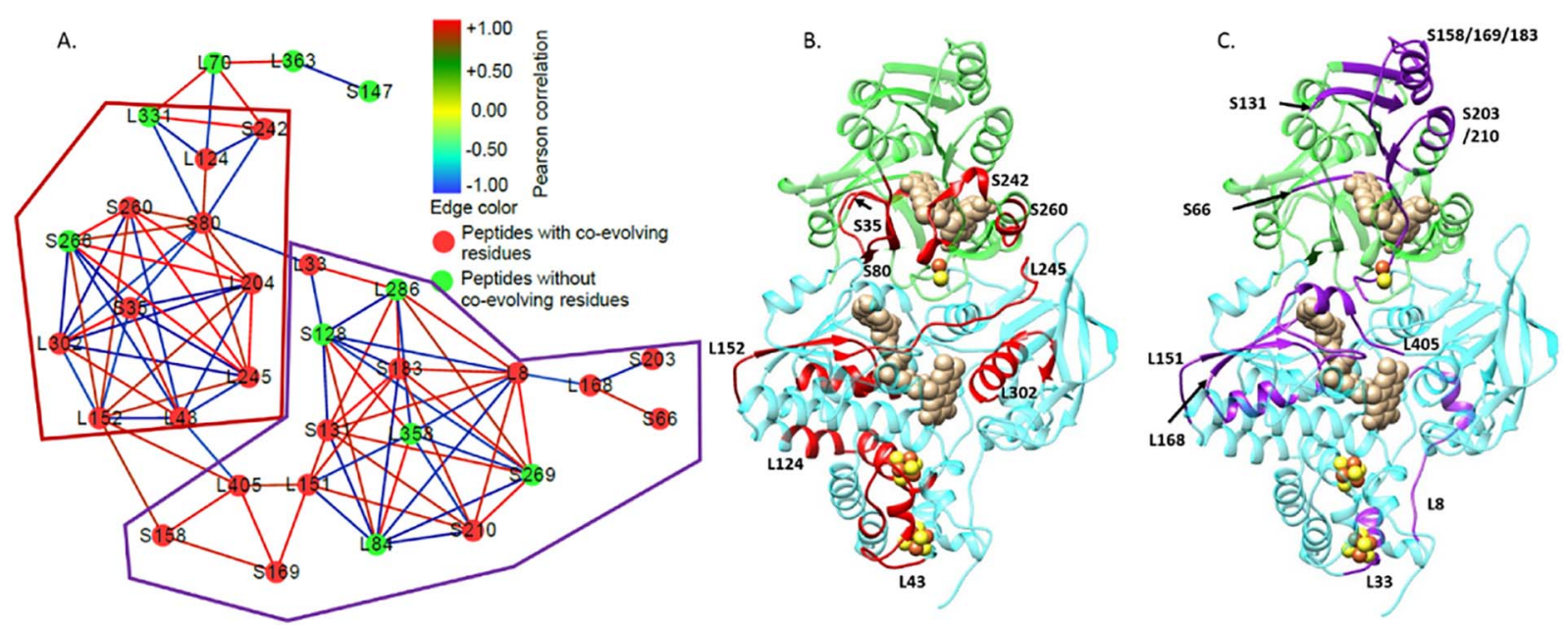

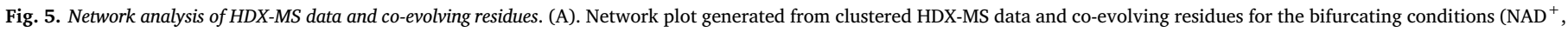

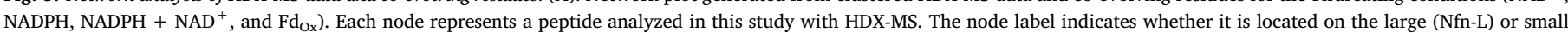

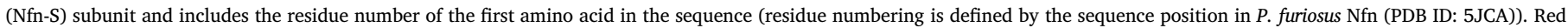

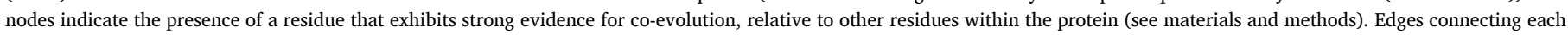

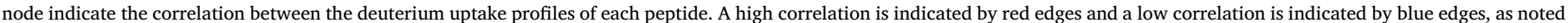

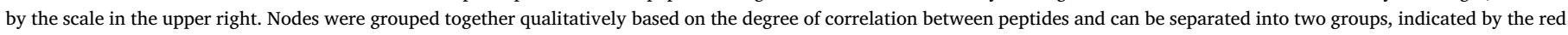

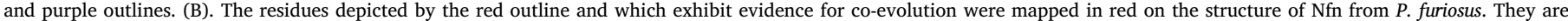

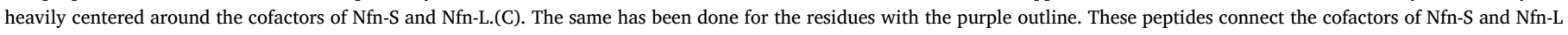
and the $\mathrm{NADH} / \mathrm{NAD}^{+}$binding site. (For interpretation of the references to colour in this figure legend, the reader is referred to the web version of this article.)

binding sites and subunits to ensure efficient function [e.g., [14,15]]. HDX-MS of $P f \mathrm{Nfn}$ in the presence of different substrates and products revealed behavior consistent with allosteric regulation (Fig. 3A-F). The goal of this experiment was to track the conformational dynamics of $\mathrm{Nfn}$ as it progresses through the catalytic cycle to elucidate the functional role of protein motion. We hypothesized that these effects are involved in tuning the affinity of Nfn for substrate, or gating the electrons by affecting the protein microenvironment.

The transfer of electrons within proteins is highly sensitive to distance, with very few examples in which there is $>14 \AA$ between sites
[28]. Most of the cofactors within Nfn are within efficient electron transfer distance of one another $(<14 \AA$ ), with the exception of the LFAD and the $[2 \mathrm{Fe}-2 \mathrm{~S}]$ cluster of Nfn-S, which are separated by a distance of $14.1 \AA$ As as revealed by the crystal structure of $P f \mathrm{Nfn}$ [4]. It was previously hypothesized that Nfn undergoes a structural rearrangement that brings the L-FAD and the [2Fe-2S] cluster of Nfn-S together during catalysis [6,29]. HDX-MS of $P f$ Nfn shows that residues $210-226$ of Nfn$\mathrm{S}$ are the most protected from deuterium exchange when $\mathrm{NAD}^{+}$and NADPH are simultaneously bound to the complex (Fig. 3B). This increase in protection is consistent with a structural rearrangement that 
brings the two cofactors closer together, improving the efficiency of electron transfer while decreasing the accessibility of several amide hydrogens to exchange. A similar effect is seen at residues 245-262 of Nfn-L, which are near the L-FAD binding site (Fig. 3C). This additional protection from deuterium exchange when $\mathrm{NAD}^{+}$and $\mathrm{NADPH}$ are present may act as a way of orienting the L-FAD closer to the [2Fe-2S] cluster of Nfn-S. Demmer et al. performed HDX-MS experiments on Nfn purified from $\mathrm{Tm}$ and hypothesized that a rigid body movement in the small subunit brings the FAD of the large subunit and [2Fe-2S] cluster of the small subunit closer together in Tm Nfn [6]. These two cofactors are $15 \AA$ apart in the crystal structure, however, upon binding of $\mathrm{NADPH}$, the distance becomes $\sim 13 \AA$ [6]. Our analysis suggests that rather than a rigid body movement, a more sophisticated means of communication likely occurs within Nfn during catalysis.

To further test our hypothesis and to supplement our understanding of how long distance communication is accomplished in Nfn, SCA was used to identify co-evolving amino acids for use in predicting a pathway of communication (Fig. 4). We identified the presence of a pathway of amino acids that connects cofactors and ligand binding sites within and between subunits, providing a potential physical conduit for information transfer through Nfn. To test whether the pathway of co-evolving residues facilitate allostery, we combined our HDX data on protein dynamics with our SCA data on co-selection (co-evolution) of residues).

The percent change in deuterium uptake for each condition was used to cluster peptides based on HDX profiles generated using early, middle, and late time points. (Supp. Figs. 1-7). By grouping peptides with similar HDX profiles under bifurcating and confurcating reaction conditions, networks were generated. These networks were then overlaid with the identity of the co-evolving residues and the extent that they are undergoing co-evolution. In the bifurcating conditions, peptides with co-evolving residues formed a sub-network together and are all highly correlated (Fig. 5A). When mapped onto the structure of Nfn, many of these peptides are localized near the cofactors of both Nfn-S and Nfn-L (Fig. 5B). The high correlation of the deuterium uptake profiles and the presence of co-evolving residues pinpoints a scaffold for communication around the cofactors involving these peptides. These subtle structural rearrangements in $\mathrm{Nfn}$ involve conformational switching at the Nfn-S and Nfn-L interface controlling the flow of electrons. A second network of peptides with correlated deuterium uptake and co-evolving residues is more distributed across Nfn (Fig. 5C). These peptides are located near the NADH/NAD ${ }^{+},[2 \mathrm{Fe}-2 \mathrm{~S}]$ cluster, and S-FAD binding site of Nfn-S, and the L-FAD binding site, as well as the interface between Nfn-L and Nfn-S. This pathway suggests the presence of a signaling network throughout the complex that could function as a sensor for pyridine nucleotides.

In this study we have made progress toward revealing the finer mechanistic details of FBEB by using HDX-MS and SCA techniques to reveal multiple pathways of communication within Nfn purified from $P$. furiosus. Our analysis of $P f \mathrm{Nfn}$ suggests that $\mathrm{Nfn}$ is a precisely tuned catalyst that undergoes coordinated motions using networks of amino acids. We hypothesize that the connectivity illustrated by these networks allows Nfn to integrate input from multiple substrate and product concentrations making it an important factor in the gating of electrons. It was previously hypothesized that electron gating in Nfn occurred through a rigid body movement [6]. Our in-depth analysis on the conformational dynamics of Nfn revealed physical networks that could utilize allosteric communication to trigger the conformational gating of electrons. Additionally the HDX-MS data collected on Nfn here suggests that a network of peptides displaying similar exchange behavior, that also contain co-evolving residues, are responsible for coordinating sophisticated movements within the complex upon nucleotide substrate and Fd binding. This suggests that the physical connectivity of the peptides plays a role in allostery, and helps the complex prepare to send electrons in either a bifurcating or confurcating direction. The connectivity of peptides also influences the gating of electrons, ensuring that an electron transfers down both the high and low potential pathways. Additionally, it could also play a role in the transfer of protons, which occurs simultaneously with electron transfer during bifurcation [4]. With the identification of key amino acids involved in communication within $\mathrm{Nfn}$, we can now focus our efforts on determining the specific role of each amino acid residue in the FBEB mechanism.

\section{Conflict of interest}

The authors declare that they have no conflicts of interest with the contents of this article.

\section{Transparency document}

The http://dx.doi.org/10.1016/j.bbagen.2017.10.002 associated with this article can be found, in online version.

\section{Acknowledgements}

This work is supported as part of the Biological and Electron Transfer and Catalysis (BETCy) EFRC, an Energy Frontier Research Center funded by the U.S. Department of Energy, Office of Science (DESC0012518). We would like to thank Montana State University Microfabrication Facility for help in preparation of gold-coated borosilica capillaries for non-covalent mass spectrometry. The Mass Spectrometry Facility at MSU is supported in part by the Murdock Charitable Trust and an NIH IDEA program grant P20GM103474.

\section{Appendix A. Supplementary data}

Supplementary data to this article can be found online at https:// doi.org/10.1016/j.bbagen.2017.10.002.

\section{References}

[1] W. Buckel, R.K. Thauer, Energy conservation via electron bifurcating ferredoxin reduction and proton/ $/ \mathrm{Na}^{+}$translocating ferredoxin oxidation, BBA-Bioenergetics 1827 (2013) 94-113.

[2] G. Herrmann, E. Jayamani, G. Mai, W. Buckel, Energy conservation via electrontransferring flavoprotein in anaerobic bacteria, J. Bateriol. 190 (2008) 784-791.

[3] J.K. Demmer, H. Huang, S. Wang, U. Demmer, R.K. Thauer, U. Ermler, Insights into Flavin-based FBEB via the NADH-dependent reduced ferredoxin: NADP oxidoreductase structure, J. Biol. Chem. 290 (2015) 21985-21995.

[4] C.E. Lubner, D.P. Jennings, D.W. Mulder, G.J. Schut, O.A. Zadvornyy, J.P. Hoben, M. Tokmina-Lukaszewska, L. Berry, D.M. Nguyen, G.L. Lipscomb, B. Bothner, A.K. Jones, A. Miller, P.W. King, M.W.W. Adams, J.W. Peters, Mechanistic insights into enegy conservation by flavin-based FBEB, Nat. Chem. Biol. 13 (2017) 655-659.

[5] D.M.N. Nguyen, G.J. Schut, O.A. Zadvornyy, M. Tokmina-Lukaszewska, S. Poudel, G.L. Lipscomb, L.A. Adams, J.T. Dinsmore, W.J. Nixon, E. Boyd, B. Bothner, J.W. Peters, M.W.W. Adams, Two functionally distinct bifurcating NADP-dependent ferredoxin oxidoreductases maintain the primary redox balance of Pyrococcus furiosus, J. Biol. Chem. 292 (2017) 14603-14616.

[6] J.K. Demmer, F.A. Rupprecht, M.L. Eisinger, U. Ermler, J.D. Langer, Ligand binding and conformational dynamics in a flavin-based electron-bifurcating enzyme complex revealed by hydrogen-deuterium exchange mass spectrometry, FEBS Lett. 590 (2016) 4472-4479.

[7] J.K. Hurley, R. Morales, M. Martinez-Julvez, T.B. Brodie, M. Medina, C. GomexMorena, G. Tollin, Structure-function relationships in Anabaena ferredoxin/ ferredoxin:NADP ${ }^{+}$reductase electron transfer: insights from site-directed mutagenesis, transient absorption spectroscopy and X-ray crystallography, BBA 1554 (2002) 5-21.

[8] N.P. Chowdhury, A.M. Mowafy, J.K. Demmer, V. Upadhyay, S. Koelzer, E. Jayamani, J. Kahnt, M. Hornung, U. Demmer, U. Ermler, W. Buckel, Studies on the mechanism of FBEB catalyzed by electron transferring Flavoprotein (Etf) and Butyryl-CoA dehydrogenase (Bcd) of Acidaminococcus fermentans, J. Biol. Chem. 289 (2014) 5145-5157.

[9] V. Katta, B.T. Chait, Conformation changes in proteins probed by hydrogen-exchange electrospray-ionization mass spectrometry, Rapid Commun. Mass Spectrom. 5 (1991) 214-217.

[10] L. Konermann, J. Pan, Y. Liu, Hydrogen exchange mass spectrometry for studying protein structure and dynamics, Chem. Soc. Rev. 40 (2011) 1224-1234.

[11] S.R. Marcsisin, J.R. Engen, Hydrogen exchange mass spectrometry: what is it and what can it tell us? Anal. Bioanal. Chem. 397 (2010) 967-972.

[12] J. Rumi-Masante, F.I. Rusinga, T.E. Lester, T.B. Dunlap, T.D. Williams, A.K. Dunker, D.D. Weis, T.P. Creamer, Structural basis for activation of Calcineurin by 
Calmdoulin, J. Mol. Biol. 415 (2012) 307-317.

[13] J.R. Engen, T.E. Wales, S. Chen, E.M. Marzluff, K.M. Hassell, D.D. Weis, T.E. Smithgall, Partial cooperative unfolding in proteins as observed by hydrogen exchange mass spectrometry, Int. Rev. Phys. Chem. 32 (2013) 96-127.

[14] N.M. Goodey, S.J. Benkovic, Allosteric regulation and catalysis emerge via a common route, Nat. Chem. Biol. 4 (2008) 474-482.

[15] J.F. Swain, L.M. Gierasch, The changing landscape of protein allostery, Cur. Op, Struc. Biol, vol. 16, 2006, pp. 102-108.

[16] S.W. Lockless, R. Ranganathan, Evolutionary conserved pathways of energetic connectivity in protein families, Science 286 (1999) 295-299.

[17] N. Halabi, O. Rivoire, S. Leibler, R. Ranganathan, Protein sectors: evolutionary units of three-dimensional structure, Cell 138 (2009) 774-786.

[18] M. Luo, R. Jackson, S. Denny, M. Tokmina-Lukaszewska, K. Maksimchuk, W. Lin, B. Bothner, B. Wiedenheft, C. Beisel, The CRISPR RNA-guided surveillance complex in Escherichia coli accommodates extended RNA spacers, Nucleic Acids Res. 44 (15) (2016) 7385-7394.

[19] M.J. Chalmers, B.D. Pascal, S. Willis, J. Zhang, S.J. Iturria, J.A. Dodge, P.R. Griffin, Methods for the analysis of high precision differential hydrogen-deuterium exchange data, Int. J. Mass Spectrom. 302 (2011) 59-68.

[20] F. Sievers, A. Wilm, D. Dineen, T.J. Gibson, K. Karplus, W.Z. Li, R. Lopez, H. McWilliam, M. Remmert, J. Soding, J.D. Thompson, D.G. Higgins, Fast, scalable generation of high-quality protein multiple sequence alignments using Clustal
Omega, Mol. Syst. Biol. 7 (2011).

[21] E.F. Petterson, T.D. Goddard, C.C. Huang, G.S. Couch, D.M. Greenblatt, E.C. Meng, T.E. Ferrin, UCSF Chimera-a visualization system for exploratory research and analysis, J. Comput. Chem. 25 (2004) 1605-1612.

[22] P. Shannon, A. Markiel, O. Ozier, N.S. Baliga, J.T. Wang, D. Ramage, N. Amin, B. Schwikowski, T. Ideker, Cytoscape: a software environment for integreated models of biomolecular interaction networks, Genome Res. 13 (2003) 2498-2504.

[23] M.E. Smoot, K. Ono, J. Ruscheinski, P. Wang, T. Ideker, Cytoscape 2.8: new features for data integration and network visualization, Bioinformatics 27 (2011) 431-432.

[24] Y. Assenov, F. Ramirez, S. SChelhorn, T. Lengauer, M. Albrecht, Computing topological parameters of biological networks, Bioinformatics 24 (2008) 282-284.

[25] J.K. Hilmer, A. Zlotnick, B. Bothner, Conformational equilibria and rates of localized motion within Hepatitis B virus capsids, J. Mol. Biol. 375 (2008) 581-594.

[26] S.S. Jaswal, Biological insights from hydrogen exchange mass spectrometry, BBA Proteins Proteomics 1834 (2013) 118-1201.

[27] J.R. Engen, Analysis of protein conformation and dynamics by hydrogen/deuterium exchange MS, Anal. Chem. 81 (2009) 7870-7875.

[28] C.C. Page, C.C. Moser, P.L. Dutton, Mechanism for electron transfer within and between proteins, Curr. Op, Chem. Biol, vol. 7, 2003, pp. 551-556.

[29] J.W. Peters, A. Miller, A.K. Jones, P.W. King, M.W.W. Adams, FBEB, Curr. Op. in Chem. Biol. 31 (146-152) (2016) 6-152. 